Yervant Terzian, Daniel Weedman, Edward Khachikian, eds.

\title{
A New Numerical Method for Investigation of Evolution of Stars and Active Galactic Nuclei
}

\author{
Avetis Abel Sadoyan, Hovik Grigoryan and Gevorg Pogosyan \\ Yerevan State University, Yerevan, Armenia
}

We consider the problem of evolution for spherically symmetric configurations in the framework of Einstein theory. There are two independent functions that describe the "inner state" evolution of a configuration. In order to have an easy transformation to the Newtonian limit, where the problem of evolution is investigated, let us take as the basic functions density $\rho(t, r)$ and velocity $w(t, r)$ where $r$ is the radius and $t$ is time in the framework of the observer at the centre of the star. We write the equations of hydrodynamics in Einstein theory in a Schwarzschild coordinate system:

The equation of continuity

$$
\rho_{, t}=-\frac{e^{\Phi-\Psi}}{1-w^{2} c^{2}}\left[\rho_{, r} w\left(1-c^{2}\right)+(\rho+P)\left(w_{, r}+w\left(\frac{2}{r}-\frac{4 \pi(\rho+P) r^{2}}{r-2 m}\right)\right)\right]
$$

and the Newton-Euler equation

$$
w(t, r)_{, t}=\frac{e^{\Phi-\Psi}}{1-w^{2} c^{2}}\left[\frac{-\rho_{, r} c^{2}}{(\rho+P) \gamma^{4}}-w\left(1-c^{2}\right) w_{, r}+\left[\left(\frac{2}{r}+\chi\right) w^{2} c^{2}-\xi\right] \gamma^{-2}\right]
$$

where

$$
\begin{aligned}
\chi & =\frac{m-4 \pi \rho r^{3}}{r(r-2 m)}, \\
\xi & =\frac{m+4 \pi P r^{3}}{r(r-2 m)},
\end{aligned}
$$

and $w=V e^{\Phi(t, r)-\Psi(t, r)}$ is the velocity of matter in the comoving frame, while $V$ is the derivative of radius with respect to time, $\gamma=\left(1-w^{2}\right)^{\frac{1}{2}}$ is the coefficient of relativity; it is one when the velocities are very small compared with the speed of light. The derivatives of a function with respect to time $t$ and radius $r$ are denoted as $f_{, t}$ and $f_{, r}$ respectively. Here we use a unit system, where the gravitational constant and the speed of light are equal to one. $P$ is pressure on the sphere (remember that we consider spherical symmetry), $c$ is the speed of sound in matter, $e^{2 \Phi(t, r)}=g_{00}, e^{-2 \Psi(t, r)}=g_{11}$, are metric coefficients, $m(r, t)$ is the accumulated mass inside the radius $r$.

These equations come to Oppenheimer - Volkoff equations when one takes $w=0$ (Oppenheimer \& Volkoff 1938).

The equation of state is assumed to be given in the form $P=P(\rho)$ for degenerate stellar matter, and no other limitations are used. 
As we see, there are two additional functions $m(t, r)$ and $\Phi(t, r)-\Psi(t, r)$. We use two of the remaining 4 equations from the set of Einstein's equations and obtain

$$
\begin{gathered}
m(t, r)=\int 4 \pi T_{0}^{0} r^{2} d r=\int 4 \pi r^{2} \frac{\rho+P w^{2}}{1-w^{2}} d r \\
\frac{\partial \Phi(t, r)}{\partial r}-\frac{\partial \Psi(t, r)}{\partial r}=\frac{4 \pi(P-\rho) r^{2}}{r-2 m}+\frac{2 m}{r(r-2 m)}
\end{gathered}
$$

An algorithm and numerical code are developed for the solution of this system of equations and the time evolution of stellar bodies is investigated (Grigoryan, Sadoyan, 1994) for model examples.

The numerical integration is based on the method of characteristics, a more accurate and reliable method than the method of finite differences or spectral methods. The main advantage of this method is that there is no need to use sophisticated approaches such as calculations "against the wind" or introducing artificial viscosity, because it contains itself defence from such types of difficulties; the only weak point is the catastrophic increase of errors as have all other methods. We have developed some subroutines for special cases, for example for slow rotation of spherically symmetric superdense bodies in the frame of Einstein theory ( A. Sadoyan, 1998$)$ in $\Omega(t, r)$ approximation $(\Omega$ is the angular velocity of rotation). This approximation corresponds to spherical rotation with Coriolis forces taken into account .

Also a subroutine for investigation of shocks waves is established that can trace the origin, propagation and decease of shocks. We expect shocks in stellar evolution processes when other methods couldn't handle the situation.

Now we are looking for observational data concerning evolution of celestial bodies for the method developed and we hope that this new method can help to understand dynamical processes in Active Galactic Nuclei.

\section{References}

Oppenheimer, J. R., \& Volkoff, G. M. 1938, Phys.Rev, 55, 374

Grigoryan, H., Sadoyan, A. 1994, Astrofizika, 37, 671

Sadoyan, A. 1997, in Abstracts of Plenary Lectures and Contributed Papers at General Relativity 15 meeting, Pune. 